\title{
Formación de maestros enfocada a la construcción de paz desde el reconocimiento y la justicia social*
}

\author{
Jenny Johanna Ruíz Peña* \\ Mirella Martínez Ramos*** \\ Diana Magnolia Burgos Méndez****
}

Recibido: 30 de enero de 2017 - Aprobado: 24 de abril de 2017

\section{Resumen}

La educación colombiana busca desarrollar un enfoque diferencial basado en el mejoramiento de los procesos institucionales. Se sistematiza la experiencia "Sujetos constructores de convivencia pacífica desde el reconocimiento", aplicada en la Escuela Normal Superior de Saboyá (Boyacá, Colombia), cuyo objetivo fue la resignificación de la educación integrando la justicia social, aspecto que fortalece el reconocimiento de los estudiantes como sujetos de derechos en la cotidianidad de la vida escolar y en el desarrollo de las competencias propias de la formación de maestros. Esta iniciativa analiza las características del contexto educativo, diagnosticando situaciones conflictivas que generan modos de menosprecio expuestos en la teoría de Axel Honnet (2010), y las formas de reconocimiento que se tejen en la interacción de los estudiantes en su entorno educativo y que nos permiten transformar dichas problemáticas desde las vivencias directas de los actores involucrados. Este artículo busca recuperar el protagonismo de la escuela en la formación de las futuras generaciones para no repetir los ciclos de violencia en el posconflicto colombiano, haciendo énfasis en el reconocimiento, porque es desde allí que se construyen y se reconfiguran los procesos sociales que contribuyen a la formación de una cultura de paz basada en la justicia social.

Palabras clave: construcción de paz; educación; justicia social; sistematización de experiencia y reconocimiento

\footnotetext{
* Artículo de investigación. Esta experiencia surge a partir de un diagnóstico realizado a la comunidad educativa que evidenciaba el aumento del conflicto escolar, el cual se mostraba en conductas de maltrato, abuso de poder, agresividad y diversos modos de menosprecio que requerían de la búsqueda de estrategias pedagógicas orientadas a reconocimiento de los estudiantes como sujetos de derechos, además de lograr su participación directa en la eliminación de dichas conductas, todo ello para propiciar la convivencia pacífica, la participación democrática y la construcción de paz. DOI: http://dx.doi.org/10.15332/s0120-8454.2017.0091.05

** Especialista en Pedagogía de la Recreación Ecológica. Estudiante de Maestría en DD. HH., Universidad Pedagógica y Tecnológica de Colombia. Correo electrónico: jennyruizp@gmail.com. Vereda Resguardo, Saboyá, Boyacá, Colombia. Teléfono: (098)7255146. ORCID: http://orcid.org/0000-0003-2582-1617

*** Especialista en Pedagogía de la Recreación Ecológica. Estudiante de Maestría en DD. HH., Universidad Pedagógica y Tecnológica de Colombia. Correo electrónico: mimarte08@hotmail.com. Vereda Resguardo, Saboyá, Boyacá, Colombia. Teléfono:(098)7255146. ORCID: http://orcid.org/0000-0003-2582-1617

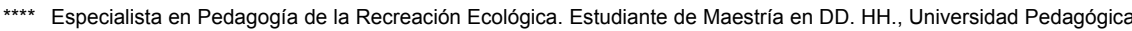
y Tecnológica de Colombia. Correo electrónico: dianamagnolis@gmail.com Vereda Resguardo, Saboyá. Boyacá, Colombia. Teléfono: (098)7255146. ORCID: http://orcid.org/0000-0003-2582-1617
} 


\title{
Teachers training focused on the construction of peace from the recognition and social justice*
}

\author{
Jenny Johanna Ruíz Peña** \\ Mirella Martínez Ramos ${ }^{* * *}$ \\ Diana Magnolia Burgos Méndez
}

Abstract

Colombian education seeks to develop a differential approach based on the improvement of institutional processes. The experience "Constructive subjects of peaceful coexistence from the recognition" was systematized. This experience was applied in the Normal Superior School of Saboyá (Boyacá, Colombia), whose objective was the resignificación of education integrating social justice, an aspect that strengthens the recognition of the students as subjects of rights in the daily life of the school life and in the development of the competences of the teachers training. This initiative analyzes the characteristics of the educational context, determining conflictive situations that generate modes of contempt presented in the theory of Axel Honnet (2010), and the forms of recognition that appear in the interaction of students in their educational environment and allow us to transform these problems from the direct experiences of the actors involved. This article pretends to recover the protagonism of the school in the formation of future generations in order to avoid repeating the cycles of violence in Colombian postconflict, emphasizing recognition because it allows that social processes grow and contribute to create a culture of peace based on social justice.

Keywords: education; peacebuilding; social justice; systematization of experience and recognition

* Research article. This experience arises from a diagnosis made to the educational community that evidenced the increase of the school conflict, which was shown in behaviors of mistreatment, abuse of power, aggression and different forms of disdain that required the search of pedagogical strategies oriented to the acknowledgement of students as subjects of rights, in addition to achieving their direct participation in the elimination of such conduct, all to promote peaceful coexistence, democratic participation and peace building. DOI: http://dx.doi.org/10.15332/s0120-8454.2017.0091.05

** Specialist in Pedagogy of Ecological Recreation. Student of the Master's Degree in Human Rights, Pedagogical and Technological University of Colombia. Email: jennyruizp@gmail.com. Vereda Resguardo, Saboya, Boyacá, Colombia. Phone: (098) 7255146. ORCID: http://orcid.org/0000-0003-2582-1617

*** Specialist in Pedagogy of Ecological Recreation. Student of the Master's Degree in Human Rights Pedagogical and Technological University of Colombia. Email: mimarte08@hotmail.com. Vereda Resguardo, Saboya, Boyacá, Colombia. Phone: (098) 7255146. ORCID: http://orcid.org/0000-0003-2582-1617

**** Specialist in Pedagogy of Ecological Recreation. Student of the Master's Degree in Human Rights, Pedagogical and Technological University of Colombia. E-mail: dianamagnolis@gmail.com Vereda Resguardo, Saboya. Boyacá, Colombia. Phone: (098) 7255146. ORCID: http://orcid.org/0000-0003-2582-1617 


\title{
Formation des enseignants axée sur la construction de la paix à partir de la reconnaissance et de la justice sociale*
}

\author{
Jenny Johanna Ruíz Peña** \\ Mirella Martínez Ramos*** \\ Diana Magnolia Burgos Méndez ${ }^{* * *}$
}

\section{Résumé}

L'éducation colombienne cherche à développer une approche différentielle centrée sur l'amélioration des processus institutionnels.On sytèmatise l'expérience «Sujets constructeurs de coextience pacifique à partir de la reconnaissance », appliquée à l'École Normale Supérieure de Saboyá (Boyacá, Colombie), dont le but était de resignifier l'éducation à partir de l'intégratoin de la justice sociale, aspect qui renforce la reconaissance des étudiants comme sujets de droits dans la vie scolaire quotidienne et dans le développement des compétences propres de la formation des enseignants. Cette initiative analyse les caractéristiques du contexte éducatif, à travers le diagnostic de situations conflictives qui génèrent des formes de mépris exposés dans la théorie d'Axel Honnet (2010), et les formes de reconnaissance qui se tissent dans l'interaction des élèves dans leur environnement d'apprentissage et qui nous permettent de transformer ces problèmes à partir des expériences directes des acteurs concernés. Cet article vise à récupérer le rôle de l'école dans la formation des générations futures pour ne pas répéter les cycles de violence dans le post-conflit colombien, en insistant sur la reconnaissance, parce que c' est à partir de là que se construisent et se reconfigurent les processus sociaux qui contribuent à la formation d'une culture de paix fondée sur la justice sociale.

Mots clés: construction de paix; éducation; justice sociale; sistematisation d'expérience et reconnaissance

\footnotetext{
* Article de recherche. Cette expérience naît d'un diagnostic fait à la communauté éducative qui a révélé une augmentation du conflit scolaire, lequel se manifestait à travers de comportement abusif, abus de pouvoir, agressivité et différents modes de mépris qui exigaient la recherche de stratégies pédagogiques visant à la reconnaissance des étudiants en tant que sujets de droits et d'obtenir leur participation directe dans l'élimination de ces comportements, tout cela pour promouvoir la coexistence pacifique, la participation démocratique et consolidation de la paix. DOI: http://dx.doi. org/10.15332/s0120-8454.2017.0091.05

** Spécialiste en Pédagogie de la Récréation Écologique. Étudiant de la Maîtrise en Droits de I'Homme, Université Pédagogique et Technologique de Colombie. Email: jennyruizp@gmail.com. Vereda Resguardo (Saboyá. Boyacá). Téléphone: (098)7255146. ORCID: http://orcid.org/0000-0003-2582-1617

*** Spécialiste en Pédagogie de la Récréation Écologique. Étudiant de la Maîtrise en Droits de l'Homme, Université Pédagogique et Technologique de Colombie. Email: mimarte08@hotmail.com. Vereda Resguardo (Saboyá. Boyacá). Téléphone: (098)7255146. ORCID: http://orcid.org/0000-0003-2582-1617

**** Spécialiste en Pédagogie de la Récréation Écologique. Étudiant de la Maîtrise en Droits de I'Homme, Université Pédagogique et Technologique de Colombie.Email: dianamagnolis@gmail.com. Vereda Resguardo (Saboyá. Boyacá). Téléphone: (098)7255146. ORCID: http://orcid.org/0000-0003-2582-1617
} 


\section{Introducción}

La educación de hoy busca que la escuela, más allá de ser un verdadero espacio de adquisición y producción de saberes, ofrezca un ambiente sano, de convivencia justa y democrática, que permita que los estudiantes se formen dentro de un entorno que les brinde las condiciones necesarias para su desarrollo personal, social, emocional y cognitivo, que legitime el reconocimiento como derecho y expresión de justicia social.

El carácter agresivo, violento e intolerante que parece ir en aumento en la interacción entre los estudiantes de las escuelas se ha convertido en un asunto importante para la investigación y la política pública, teniendo en cuenta su incidencia en el desarrollo social, en el desempeño y los logros académicos de los niños, niñas y jóvenes en formación y en el clima institucional (MartínezOtero, 2005, p. 4).

Las instituciones educativas reflejan el accionar de la sociedad de la cual hacen parte, son el epicentro de un sinnúmero de culturas, vivencias, personalidades, expectativas e intereses, entre otros aspectos propios de la singularidad del ser humano (Gobernación del Huila, 2015). La Escuela Normal Superior de Saboyá (ENSSA) se ha propuesto ser pionera de cambios significativos de orden social a través de acciones pedagógicas que vayan modificando progresivamente la mentalidad y conducta de los integrantes de la comunidad educativa, y a través de esta, la comunidad en general. De ahí que haya puesto especial énfasis en una educación más humana, que permita la inclusión, cuyo componente más importante sea el reconocimiento como base de una educación integral, contrarrestando los modos de menosprecio a los cuales se enfrentan los estudiantes en su interacción con el entorno escolar por motivos de género, raza, edad, religión, cultura o diversidad funcional

La experiencia se desarrolló con los estudiantes de los grados cuarto y quinto de básica primaria, con los estudiantes de la educación básica secundaria, la educación media y del Programa de Formación Complementaria, quienes hicieron parte del proyecto en las etapas de diagnóstico, intervención y evaluación; posteriormente se convirtieron en pares que replicaron las experiencias y conocimientos. Los directivos y docentes de la institución, el orientador escolar, los padres de familia y algunas entidades gubernamentales de orden municipal dieron su apoyo incondicional y permanente, reconociendo que las dinámicas propias de la educación tienen que ver con el trato digno y la construcción de situaciones que coadyuvan al respeto por la persona durante el encuentro educativo y que permea las demás dimensiones de orden social.

La experiencia representó una resignificación del proyecto educativo institucional (PEI) y del sistema de evaluación, permitió introducir aspectos más incluyentes y formativos que realmente atiendan al estado de vulnerabilidad de los niños, niñas y jóvenes. También fue necesario el fortalecimiento del acuerdo de convivencia desde el ejercicio de los derechos y la justicia social, con un debido 
proceso formativo-pedagógico desde la perspectiva del derecho a la educación y desarrollo humano, buscando que la resolución pacífica de los conflictos potencie la construcción de convivencia democrática, la reconstrucción del tejido social y la tan anhelada paz que requiere el país.

Los efectos anteriores muestran: 1) que sí es posible pensar la educación desde, con y para la justicia social y asegurar ambientes de equidad donde las desigualdades sean el motivo central de inclusión, consenso y oportunidad para hacer visibles las realidades ocultas tradicionalmente; 2) que es necesario visibilizar acciones de reconocimiento entre los integrantes de la comunidad educativa como medio para la construcción de una convivencia pacífica, superando diferentes modos de menosprecio y humillación presentes en las interrelaciones.

Las escuelas normales sí pueden ayudar a transformar las realidades y hablar de manera esperanzadora, porque los seres humanos no toda la vida hemos sido violentos ni tampoco estamos condenados a la violencia; 3) que sí es posible educar las nuevas generaciones en el manejo de conflictos, conscientes de que la paz no es solamente ausencia de estos, sino la capacidad para administrarlos y resolverlos de forma adecuada; 4) que la ENSSA, como formadora de maestros, crea espacios de debate, reflexión y formación para la convivencia pacífica, fundados en el reconocimiento de sí mismos y del otro como sujeto de derechos, con lo cual promueve la justicia social.

Las escuelas normales, como instituciones formadoras de maestros y maestras, tienen un papel importante en el proceso inicial de consolidación de la paz, porque son ellos y ellas quienes en gran parte del territorio nacional forman individuos capaces de comprender la realidad social de sus comunidades y son quienes están llamados(as) a juzgar menos y comprender más, a dar ejemplo de reconciliación como vía para superar el odio y parar una guerra que empieza en las palabras y no termina destruyendo al otro, sino que ayuda a sacar lo mejor de la persona que lo siente.

La exposición de la experiencia cuyas motivaciones, efectos y certezas se acaban de presentar, comprende, primero, la descripción y diagnóstico de la situación de la Escuela Normal Superior de Saboyá desde la justicia social, el reconocimiento y modos de menosprecio en las relaciones que se dan entre los niños, niñas y jóvenes; segundo, una aproximación teórica a las categorías de reconocimiento, menosprecio y justicia social a nivel educativo; en un tercer aparte se explica la metodología de la experiencia "Sujetos constructores de convivencia pacífica desde el reconocimiento"; en un cuarto acápite se presentan los principales resultados encontrados y las proyecciones con relación a los procesos de construcción de paz en el ámbito educativo, y en el punto quinto las conclusiones.

Los resultados relacionados con la construcción de paz en la institución educativa confirman que la escuela es el mejor espacio para el restablecimiento del tejido social y un motor importante respecto al cambio que el país necesita, porque al implementar estrategias pedagógicas que promuevan la convivencia 
pacífica y las formas de reconocimiento emocional, jurídico y social se garantiza la práctica de los derechos humanos, permitiendo contrarrestar los modos de menosprecio presentes en la cotidianidad de la escuela y su entorno.

\section{La Escuela Normal Superior de Saboyá (Boyacá)}

La institución educativa Escuela Normal Superior (ENSSA) se encuentra ubicada en el municipio de Saboyá, departamento de Boyacá, una región de origen campesino, de gente trabajadora, dedicada a la agricultura -cultiva especialmente maíz y papa-y a la ganadería -ganado bovino, en su mayoría-. La ENSSA surgió para dar respuesta a una necesidad sentida del municipio y especialmente de la región: la formación de sus niños, niñas y jóvenes. Cuenta con 17 sedes ubicadas en la zona rural y dos sedes urbanas en la cabecera municipal. Ofrece los niveles de preescolar, básica primaria, básica secundaria, media y formación complementaria (Escuela Normal Superior de Saboya, 2016).

En sus 61 años de trayectoria en la formación de docentes, se ha convertido en un eje de constante progreso intelectual, económico, social, político y cultural, así como de desarrollo de la región y del país. Los profesionales que ha educado se distinguen por dejar huella en diferentes espacios de la vida nacional, contribuyendo desde su labor pedagógica con el cambio de la actitud violenta de la comunidad, por una mentalidad pacífica y de convivencia social (Escuela Normal Superior de Saboya, 2016).

En la actualidad la ENSSA es reconocida a nivel municipal y departamental como una de las instituciones formadoras de maestras y maestros en preescolar y básica primaria; por prepararlos como personas críticas e innovadoras en pedagogía y didácticas contemporáneas, a través del desarrollo de proyectos y programas de formación complementaria, y por ofrecer a los estudiantes una educación integral.

En el año 2013, la institución hizo parte del proyecto denominado Formación de Maestros para una educación como derecho con justicia social en las Escuelas Normales Superiores, dirigido por la Universidad Pedagógica Nacional, orientado a actualizar a algunos docentes, quienes elaboraron un diagnóstico para reconocer situaciones de justicia e injusticia en las cuales se veían inmersos los estudiantes y favorecían episodios de menosprecio -como el maltrato físico y verbal, ofensas entre pares, discriminación y humillaciones por su condición social, religiosa y de género- con el fin de llevar a cabo un acompañamiento que permitiera tener una visión holística que garantizará una sociedad más justa, con mejores oportunidades para todos, propiciando ambientes de convivencia pacífica.

Según los informes comparativos en materia de manejo de conflictos y casos difíciles, años 2012-2015, se encontró la siguiente situación en relación con la violencia escolar: 
Tabla 1. Casos relacionados con la violencia escolar entre los estudiantes de la I.E.

\begin{tabular}{|c|c|c|c|c|}
\hline Año & 2012 & 2013 & 2014 & 2015 \\
\hline Casos & 92 & 112 & 147 & 38 \\
\hline
\end{tabular}

Fuente: Informe del índice de violencia escolar comparativo. ENSSA, 2015.

Como se observa, en los años 2013 y 2014 se presentó la mayor cantidad de casos, lo cual coincide con la implementación de la experiencia de paz "Sujetos constructores de convivencia pacífica desde el reconocimiento" ${ }^{13}$ y su repercusión en los estudiantes, ya que sintieron libertad de manifestar abiertamente situaciones en las cuales consideraban vulnerados sus derechos por parte de algunos integrantes de la comunidad educativa -como indefensión, desconfianza, inseguridad, exclusión, marginación y estigmatización-, lo cual no les permitía desarrollarse de manera integral y, por el contrario, contribuía a generar daños que quebrantaban en cierta medida la relación consigo mismos y afectaban la autoconfianza, el autorrespeto y la autoestima.

Como resultado, se determinó que la situación socioafectiva que presentan las niñas, niños y jóvenes está determinada por problemas sociales como la conformación de la familia; la mayoría de estas son disfuncionales, por tanto, se ven obligados a vivir con abuelos, tíos, primos, padrastro o madrastra u otro tipo de familiares; no hay un núcleo familiar definido que les permita tener un modelo a seguir y mayor estabilidad personal y afectiva. Muchos de los padres de estos pequeños en su niñez fueron víctimas de agresiones, maltrato físico y psicológico, discriminación y violencia por parte de sus progenitores, y algunos han repetido el ciclo vicioso de la agresión, aunado a la soledad en la que viven.

Debido a las anteriores situaciones, se reviste de importancia la teoría de Axel Honneth (2010, p. 20), según la cual el reconocimiento se configura como una de las dimensiones de la justicia social, destacando especialmente la esfera del amor, porque en esta se establecen los vínculos filiales en la familia como primer nicho social, que determinan los rasgos emocionales como las demostraciones de afecto, sentimientos positivos ligados con las relaciones basadas en el reconocimiento, el apoyo y, ante todo, el amor que se prolonga a través de las generaciones.

De igual manera, es significativo que la familia disponga de los recursos necesarios para satisfacer necesidades básicas como vivienda, nutrición, salud y educación; sin embargo, aunque importantes, estas no deben sobrepasar la esfera del amor, la cual le permite al niño configurar sus estructuras cognitivas y afectivas básicas, posibilitándole aprender a valorarse en relación con otros

13 Esta experiencia se desarrolló en el año 2013 en la Institución Educativa Escuela Normal Superior de Saboyá. Su finalidad era la búsqueda de estrategias pedagógicas, orientadas al reconocimiento de los estudiantes como sujetos de derechos y a lograr su participación directa en la eliminación de modos de humillación, agresión y vulneración de derechos, para propiciar la convivencia pacífica, la participación democrática y la construcción de equidad que se requiere en una institución formadora de maestros, quienes serán los encargados de educar la sociedad del mañana. 
seres humanos, a respetarse a sí mismo y a los demás, dignificarse como persona y mejorar las interacciones que establece a diario con los diferentes miembros de la comunidad.

\section{Aproximación teórica a las categorías de reconocimiento, menosprecio y justicia social}

\section{El reconocimiento}

Para el desarrollo de esta sistematización se tomó la teoría de las formas de reconocimiento y modos de menosprecio desarrolladas por Axel Honnet, que se convierten en elementos claves en la comprensión y análisis de la interacción que viven los niños, niñas y jóvenes a nivel personal, familiar y social relacionándolas con la agresión y los conflictos que se presentan en las aulas de clase y en el contexto escolar.

El reconocimiento para Honneth (citado en Matijasevic, 2015) se entiende como:

Un asunto clave para la identidad de los sujetos sociales históricamente situados. Además, debe ser entendido como un comportamiento - con el que respondemos de manera racional a cualidades de valor que hemos aprendido a percibir en los sujetos humanos, lo que permite a los otros identificarse con sus propias cualidades y alcanzar una mayor autonomía; diferencia así el reconocimiento del conocimiento, toda vez que este último se entiende como un acto exclusivamente cognitivo. (p. 21)

De acuerdo con la teoría de Honnet, el reconocimiento emocional, jurídico y social es el fundamento que posibilita la construcción de su propia identidad y su pleno desarrollo, estaría determinando por su autorrealización como persona, para construir la autoconfianza, el autorrespeto y la autoestima, factores que determinan la relación consigo mismo, de tal forma que evite el menosprecio, entendido como la negación del reconocimiento (maltrato físico, desposesión e injuria), categoría que está presente en las relaciones interpersonales que se dan en las instituciones educativas que registran niveles apreciables de conflicto (2010, p. 56).

El autor destaca tres formas de reconocimiento importantes en el desarrollo cultural y social de las personas, dentro de las cuales están: el reconocimiento en el amor, que hace énfasis en la individualidad de la persona, relacionado con el grupo familiar y el grupo de amigos más cercanos, donde se van creando lazos íntimos de afectividad. El reconocimiento jurídico, se refiere a la libertad que tienen las personas para desarrollarse exigiendo sus derechos y cumpliendo los deberes, independiente de las características sociales, económicas y culturales. 
La solidaridad, se refiere al reconocimiento que le hace la sociedad en la que vive, en la medida que demuestra sus habilidades, talentos y capacidades, lo cual le garantiza la valoración social y la autoestima (Honneth, 1992, 2006b).

Estas formas de reconocimiento corresponden con la realidad que viven los estudiantes en el entorno familiar, primer lugar donde se consolida la esfera del amor a partir de las expresiones de cariño, cuidado y protección, y donde se manifiestan sentimientos, se establecen lazos de comprensión, seguridad, amor y respeto por sí mismo y por el otro. La familia es el lugar privilegiado para comenzar a formar la personalidad de los niños, niñas y jóvenes, desarrollando sus habilidades y capacidades básicas para que aprendan a quererse, valorarse, a comprender su propia identidad y a confiar en sí mismos. De esta forma, aprenden también que los conflictos que se presentan en las interacciones se pueden solucionar no con la violencia, sino a partir del diálogo y la concertación de manera colectiva.

La segunda esfera, según Honneth, es el reconocimiento jurídico. Teniendo en cuenta que los niños, niñas y jóvenes son seres sociales, deben estar en condiciones de saberse sujetos de derechos, entender que son iguales a los demás y estar en capacidad de reclamarlos cuando sienten que se les vulneran. De ahí que en estos procesos de relación deban aprender unas normas básicas de convivencia que les permitan interactuar de manera adecuada con los otros. En palabras de Honneth (1992), "el deber categórico de reconocer a todos los demás la responsabilidad moral" (p.83). De esta manera los estudiantes deben asumir las responsabilidades de sus actos como personas autónomas y dignas de derechos y deberes.

En las instituciones educativas, los manuales de convivencia son el instrumento jurídico donde se establecen los acuerdos y las normas que garantizan la convivencia escolar, teniendo en cuenta los intereses, motivaciones, aportes de los estudiantes, docentes, padres de familia y demás actores de la comunidad.

En las aulas de clase, los manuales de convivencia adquieren funcionalidad en la medida en que los estudiantes conocen sus deberes, entienden las formas de relacionarse con los demás, valoran y reclaman sus derechos y reconocen al otro como un sujeto en igualdad de condiciones. Es a través de los pactos de aula donde se analizan las situaciones problémicas y se establecen acuerdos para adquirir compromisos que favorezcan la convivencia pacífica enmarcada en el buen trato, la comunicación asertiva y la toma de decisiones que contribuyan en la solución de conflictos.

La escuela, como centro de formación humana integral, debe ser un lugar seguro donde se pueda practicar y aprender a relacionarse con los otros en la diferencia, y de donde debe quedar excluida cualquier manifestación de violencia. Porque los estudiantes acuden a ella no solamente por la simple adquisición de conocimientos, sino para aprender a convivir respetando a las personas independientemente de cómo sean. 
De acuerdo a lo indicado por Arias (2016), la institución educativa tiene en cuenta algunos pactos dentro de los cuales están:

El pedagógico, se refiere a la forma como los estudiantes establecen relaciones sociales, basadas en la dignidad y los valores humanos, para su formación como ciudadanos participativos, tolerantes, innovadores, críticos, capaces de tomar decisiones que beneficien a toda la comunidad.

El didáctico, tiene en cuenta los procedimientos, métodos y estrategias que se emplean para construir y reconstruir los saberes en su relación con el entorno social y cultural.

En el político, la escuela se constituye en una pequeña sociedad en la cual se establecen las primeras estructuras jerárquicas de autoridad, que como unidad social les permitirá desarrollar diversas estrategias que favorezcan la toma de decisiones. (p. 2)

Por tanto, la esfera del reconocimiento jurídico permite a los niños, niñas y jóvenes cumplir sus deberes y obligaciones morales con la misma responsabilidad por sus acciones que los demás miembros de la comunidad educativa $\mathrm{y}$, al mismo tiempo, reconocer sus derechos como personas iguales, libres y autónomas ante los otros. Para Honnet (2010) “una generalización de la esfera del reconocimiento jurídico en la que el individuo es capaz de verse a sí mismo como persona que comparte con todos los otros miembros de su comunidad las características de un actor moralmente imputable" (p. 27).

La tercera esfera de reconocimiento, de acuerdo con Honneth, es la valoración social, o solidaridad, que constituye un factor trascendental en la formación integral de los estudiantes, pues de este depende su capacidad para estar con otros, para pasar de lo individual a lo colectivo, para entender y reconocer que los saberes que el otro posee, aunque diferentes, son valiosos y merecen todo el respeto. "Implica la posibilidad de configuración de la autoestima, que se da por aprobación solidaria y apreciación de las capacidades y formas de vida desarrolladas individualmente" (Honneth, 2010, p. 28).

De acuerdo con Honnet (citado en Díaz y González, 2015):

Esta forma de reconocimiento va más allá de la apreciación del otro como sujeto de derechos, puesto que también implica la valoración a partir de las diferencias individuales, es decir, de las cualidades que han venido configurando históricamente al otro, y que le permiten tener en cuenta sus experiencias personales y colectivas con criterios propios. (p 392)

Es importante admitir que esta forma de reconocimiento permite que los niños, niñas y jóvenes tengan un proceso de socialización más amplio que no solo incluye a su familia y al círculo de amigos, sino a un grupo más extenso 
de personas, en las cuales se generan interacciones que requieren asumir una actitud de trabajo colaborativo en búsqueda de fines comunes.

Dentro de los niños, niñas y jóvenes es común observar la capacidad que tienen para relacionarse con los otros y para establecer vínculos de amistad y de empatía a través del juego, el diálogo, el trabajo colaborativo, la participación en las clases, los eventos culturales, deportivos, recreativos, donde se evidencian sus talentos y habilidades, así como en la resolución de los conflictos que suelen presentarse en la cotidianidad. De esta manera la escuela favorece los procesos de socialización, autoestima y valoración del otro, como sujeto de posibilidades y poseedor de talentos, que merece todo el aprecio y respeto dentro del grupo. Honnet (2010) señala que "en los tres modelos de reconocimiento el amor, el derecho y la solidaridad quedan establecidas las condiciones de interacción, a partir de las cuales las personas pueden ver garantizada su dignidad o su integridad" (p. 30).

\section{El menosprecio, una forma negativa de reconocimiento}

El menosprecio, según Honnet (2010), sería la negación del reconocimiento. Se pueden encontrar, afirma el autor, tres modos de menosprecio asociados directamente con las formas de reconocimiento antes descritas: primero, la humillación o maltrato físico, que priva de la autonomía física y destruye parte de la confianza básica en el mundo y en el valor propio; segundo, la privación de derechos y la exclusión social, que implica que la persona no sea reconocida en su comunidad como un sujeto jurídico de pleno valor, alterando su autorrespeto, y tercero, el desprestigio de las formas de vida o convicciones, que lesiona el sentimiento de poseer significación social en una comunidad concreta $(p, 169)$.

Al interior de la institución educativa se presentan algunas manifestaciones de violencia a veces visibilizadas, expresadas en el maltrato físico y verbal, las cuales en Colombia están normativizadas en la Ley 1620 de 2013 (Ley de Convivencia) y en los acuerdos de convivencia, donde la institución educativa establece las rutas a seguir para evitar los diferentes modos de menosprecio que impiden el reconocimiento de derechos de los estudiantes hacia la construcción de paz basada en la justicia social.

Dentro de las aulas de clase es común encontrar situaciones de maltrato entre niños, niñas y jóvenes, evidenciadas en expresiones como amenazas, insultos, groserías, golpes, indiferencia, manifestaciones de humillación y exclusión que impiden el bienestar en la vida escolar y generan episodios de violencia que se van normalizando, afectando su dignidad.

Tovar (2003) señala que:

El maltrato provoca humillación, ya que lesiona la confianza en sí mismo y en el mundo que le rodea, siendo más destructivo en la relación 
consigo mismo que los demás modos de menosprecio y esto, en caso extremo, puede producir una muerte psíquica. (p. 4)

Por consiguiente, la falta de autoestima hace que los niños, niñas y jóvenes manifiesten más fácilmente formas de descontento, agresividad, comportamientos negativos que dañan su autoimagen y las percepciones sobre sí mismas(os), que dificultan la relación con los demás.

Siendo la escuela un espacio cultural heterogéneo donde conviven diferentes formas de pensar, actuar y sentir, se hace indispensable en este momento crear conciencia del respeto por la multiculturalidad, con el fin de que las diferencias sean una oportunidad para compartir con el otro sus saberes y no como algo que dificulte la convivencia alrededor de la institución educativa.

La escuela, entonces, se convierte en el espacio donde se aprende a convivir en la diferencia, donde se le reconoce al estudiante no solo sus conocimientos académicos, donde se generan espacios de dimensión humana que permiten relacionarse de la mejor forma con los demás. Es tarea de todos humanizar la educación con actitudes responsables de amor por el otro, en donde se dé paso al acierto y al error para responder a la necesidad de ayuda en el desarrollo personal de los demás, siendo el educador el polo de transformación de su entorno humano y profesional.

El reconocimiento que se da en contraposición a este menosprecio es el amor. A partir del amor se forma la confianza en sí mismo de carácter corporal y se validan afectos y necesidades en tanto estos son correspondidos. Solo a partir de este tipo de reconocimiento se podrán dar los demás.

El segundo modo de menosprecio es la privación de derechos o la exclusión social de un sujeto dentro de una sociedad. Este menosprecio margina y excluye socialmente a la persona, de tal forma que limita su autonomía al no poseer la igualdad de derechos socialmente válida que le permita una interacción con sus pares, propiciando la pérdida de autoestima y autorrespeto (Tovar, 2003).

En la institución educativa, los estudiantes se sienten vulnerados cuando no se respetan las normas establecidas en el Acuerdo de Convivencia; cuando no son tratados en igualdad de condiciones; cuando son excluidos por razones de raza, sexo, religión entre otros; cuando sus particularidades no son respetadas ni tenidas en cuenta por sus pares, porque se pueden estar borrando las singularidades que distinguen a unos de otros.

La situación actual del país demanda reconocer la importancia de promover las formas de reconocimiento y la justicia social en las escuelas, a través de una acción transversal e interdisciplinaria en la enseñanza, la pedagogía y el currículo, de tal forma que garantice a cabalidad los derechos de los niños y de los jóvenes, convirtiéndose en una política educativa vigente que consolide la práctica de valores y actitudes que contribuyan a la generación de una cultura de paz. 
Para Honnet (2010), el tercer tipo de menosprecio es la degradación del valor social de las formas de autorrealización. Se da cuando los sujetos no obtienen la apreciación social de aquellas capacidades que adquirieron a lo largo de su vida. El reconocimiento correspondiente a este menosprecio es la aprobación solidaria de las capacidades adquiridas individualmente. La degradación del valor social implica la falta de aceptación de la persona dentro un grupo, afectando la valoración de su propia identidad y aquello que como individuo puede aportar a los demás, o ser aceptado socialmente de acuerdo a parámetros establecidos (p. 58).

Saber convivir implica asumir los conflictos de manera asertiva, ya que forman parte de la condición humana y es preciso reconocer que estos siempre están presentes en la vida de las personas, pues atienden a la singularidad de cada uno; por tanto, se requiere aprender a interactuar en la diferencia, analizando las causas que los generan y los efectos que producen, de tal forma que prime el diálogo y la concertación colectiva para impedir se genere más violencia y se logre no solo prevenirla, sino rechazarla (Jiménez, 2012, p. 4).

\section{Aproximaciones a la justicia social}

La justicia social desde la teoría de Jhon Rawls propone la noción de justicia como un principio que regula el orden social para desarrollar la capacidad de juzgar y actuar de acuerdo a parámetros establecidos como justos, deseando que otros actúen de esta misma manera. Cada grupo de personas debe decidir cuáles serán los principios que fundamentan la sociedad en la que vive, determinando sus derechos y deberes básicos racionalmente, distinguiendo lo justo de lo injusto (1971, p. 21).

Para Rawls, la sociedad es una asociación de grupos humanos más o menos autosuficiente, dentro de la cual se crean ciertas reglas de conducta que deben cumplir sus integrantes de manera obligatoria para lograr un fin común, mediante un sistema de cooperación para hacer posible una vida mejor de lo que pudiera lograr si viviera únicamente de sus propios esfuerzos (1971, p. 18).

La teoría de la justicia social de Rawls reconoce que las instituciones presentes en la sociedad atribuyen a todos sus miembros una serie de derechos y deberes. En algunos casos las instituciones educativas favorecen ciertas costumbres que fomentan las desigualdades desde la infancia obstaculizando así sus proyectos de vida. Por lo cual es importante generar ambientes sociales equitativos que les brinden mejores condiciones a todos los integrantes de la comunidad (1971, p. 18).

\section{La justicia social en el entorno escolar}

La educación como derecho social es un indicador que permite medir el nivel de desarrollo democrático; encierra en sí misma la comprensión de la justicia social, así como el respeto a los derechos humanos. La justicia es un imperativo 
ético, político y jurídico que se sintetiza esencialmente en el terreno de las políticas sociales y educativas y en la ética de las relaciones que establecen los seres humanos en diversos campos.

Las desigualdades sociales y el fracaso escolar hacen evidente la necesidad de repensar las instituciones educativas, ya que deben ser un espacio de igualdad, equidad e inclusión atendiendo a las particularidades de los individuos en su contexto. Es en el entorno escolar donde los estudiantes manifiestan aquellas experiencias vividas en la familia como núcleo primario de formación, de tal manera que ellos se relacionan de acuerdo con el nivel de marginación social al cual han estado sometidos y cuya realidad tradicionalmente ha permanecido de cierto modo invisibilizada. Es importante ver que la educación, como derecho fundamental, se convierte en una gran oportunidad para transformar la sociedad desigual e injusta, orientando cada una de las acciones a formar estudiantes autorreflexivos y autocríticos que asuman responsabilidades individuales, grupales y sociales.

Hoy más que nunca se hace necesario hablar de justicia social en las instituciones educativas, ya que es la escuela el motor que permite generar transformaciones no solo en el aspecto educativo, sino también en ámbito cultural, económico y social. Es tarea motivar a las familias y a toda la comunidad hacia la construcción de una mejor sociedad, que evite las desigualdades y refleje acciones y comportamientos positivos a favor de la equidad escolar. Como señala Paulo Freire (citado en Melgar, 2015), "la educación no cambia el mundo: cambia a las personas que van a cambiar el mundo" (p. 10).

Enseñar en justicia social, requiere ejecutar estrategias metodológicas que permitan el cambio de actitudes y valores en el aula, no solo por parte de los estudiantes, sino del maestro que es el principal agente de transformación. La educación debe dar respuesta a los requerimientos de la sociedad actual atendiendo a sus necesidades, para que se convierta en la más poderosa herramienta en la lucha contra la deserción escolar, la exclusión, la marginación social y las desigualdades que afectan principalmente a los niños, niñas y jóvenes, quienes son el centro del quehacer educativo.

Pretender trabajar la justicia social en el campo de la educación requiere enfocarla desde una óptica de equidad, es decir, favorecer más a quienes no cuentan con las posibilidades para desarrollarse, a diferencia de otros que han tenido una gama amplia de oportunidades; por eso, la distribución igualitaria de los recursos entre los estudiantes debe compensar en mayor medida a los que no han tenido los elementos suficientes para potenciar su desarrollo integral (Antonio, 2011, p. 5).

Desde la educación se debe trabajar para no permitir la legitimización de las injusticias y las desigualdades. Por este hecho, los educadores, como líderes sociales, deben generar espacios que eviten diferentes formas de exclusión social, que favorezcan el reconocimiento de las identidades, las diferencias y la individualización, entre otros (Antonio, 2011, p. 19). 
Para garantizar una educación de calidad, Escudero y Martínez (2012) proponen un sistema educativo basado en la justicia con equidad, evitando cualquier tipo de exclusión y desigualdad, haciendo énfasis en prácticas pedagógicas de calidad por parte de los docentes, que motiven y garanticen una educación para todos, basada en el reconocimiento y evitando las diferentes formas de menosprecio (p. 178).

\section{Metodología}

La metodología de sistematización retoma la interdisciplinariedad y la integración de saberes como elementos importantes para analizar el entorno donde se desarrolló la experiencia, teniendo en cuenta las particularidades y las necesidades de quienes lo configuran.

La sistematización de la experiencia requirió recolectar información sobre las vivencias de los niños, niñas y jóvenes en su cotidianidad familiar y escolar para determinar qué tanto identificaban las categorías de reconocimiento, menosprecio y justicia social, cómo respondían a ellas y la incidencia que tuvo la estrategia en ellos.

Al efecto, se acudió a la cartografía social, como lo expresan Quintero y Rojas (2015), "la cartografía es una propuesta conceptual y metodológica que permite construir un conocimiento integral de un territorio" (p. 5). Esto permite trabajar de manera dinámica una problemática o hecho social en un contexto determinado y facilita el conocimiento integral de un territorio por parte de los miembros de la comunidad. Se toman como punto de partida representaciones graficas como dibujos, fotos y mapas, actividades que permiten a los niños, niñas y jóvenes acercarse a su espacio geográfico, socioeconómico e históricocultural de una manera didáctica e incluso lúdica, basada en la construcción siempre colectiva del conocimiento desde la participación y el compromiso social, posibilitando la transformación del mismo (Habegger y Lulia, 2007. p. 3).

La cartografía social utilizada partió de la implementación del mapa temporalsocial que reconoce los acontecimientos permanentes en la memoria del grupo objeto de estudio; para rememorar el pasado, según Quintero y Rojas (2016), los mapas temporales pretenden "comprender el presente y dibujar posibilidades futuras de actuación, que los actores pueden proponer y decidir realizar" (p. 12).

Para la sistematización de la experiencia se abordaron las siguientes etapas:

1. Fase diagnóstica: observación directa del contexto, identificando posibles acciones relacionadas con maltrato a nivel pedagógico, estilo de docencia, didáctica y evaluación. Para ello se aplicaron entrevistas semiestructuradas a los niños, niñas, jóvenes y docentes.

2. Fase de motivación y sensibilización: tuvo como fin generar en los niños, niñas y jóvenes confianza para expresar con libertad las experiencias y 
vivencias sobre las formas de reconocimiento individual y colectivo, así como las manifestaciones de menosprecio presentes en las relaciones que se establecen entre los diferentes integrantes de la institución.

3. Aplicación de la cartografía social: mediante la cual se comprenden las causas que generaron las situaciones problémicas y visibilizaron situaciones de riesgo en términos de reconocimiento y menosprecio que permitan establecer redes sociales que generen cambios positivos y duraderos en el grupo escolar, encontrando de manera colectiva algunas alternativas de transformación del entorno. De acuerdo a lo expuesto por Fernández (2007), "las redes sociales dentro de un territorio equivaldrían al conjunto de individuos que representan a organizaciones, instituciones, fundaciones, etc., los cuales establecen diversos vínculos con el fin de generar determinados tipos de relación" (p. 5).

En la implementación de la cartografía social se tuvieron en cuenta algunas etapas expuestas en el libro Desdibujando los territorios: orientación para la elaboración de cartografias sociales (Quintero y Rojas, 2016, p. 25).

Selección del tipo de mapa: se utilizó el mapa temporal-social de las formas de reconocimiento y modos de menosprecio para analizar la trayectoria en términos de pasado, presente y futuro, exponiendo las causas que han generado los conflictos entre iguales al interior de la institución educativa y que impiden el reconocimiento positivo de los estudiantes como sujetos de derecho en el núcleo familiar y escolar.

Se elaboraron dos mapas, uno de reconocimiento y otro de menosprecio, que demuestran la situación que experimentan los estudiantes a nivel familiar y escolar. Esto permitió identificar las situaciones de maltrato, humillación, violencia física y verbal que han vivido los niños, niñas y jóvenes en su proyecto de vida.

Imágenes 1a y 1b. Cartografías del pasado, presente y futuro de los niños y niñas de la ENSSA

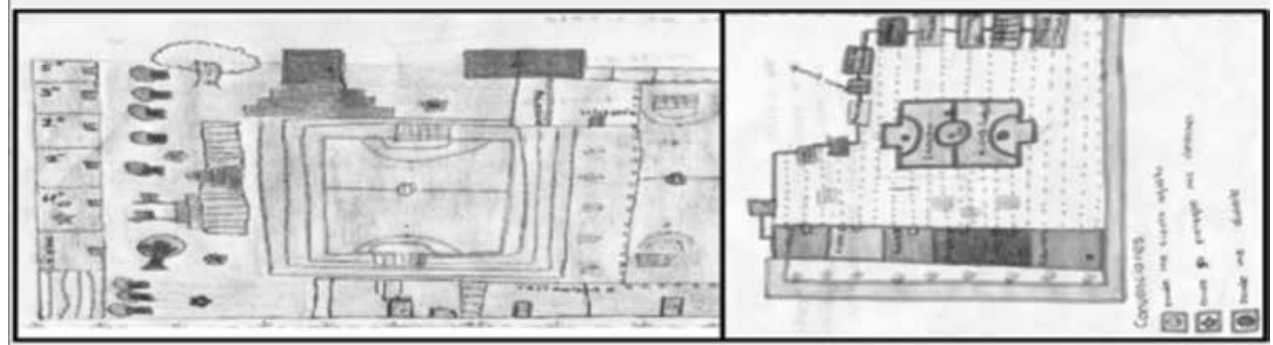




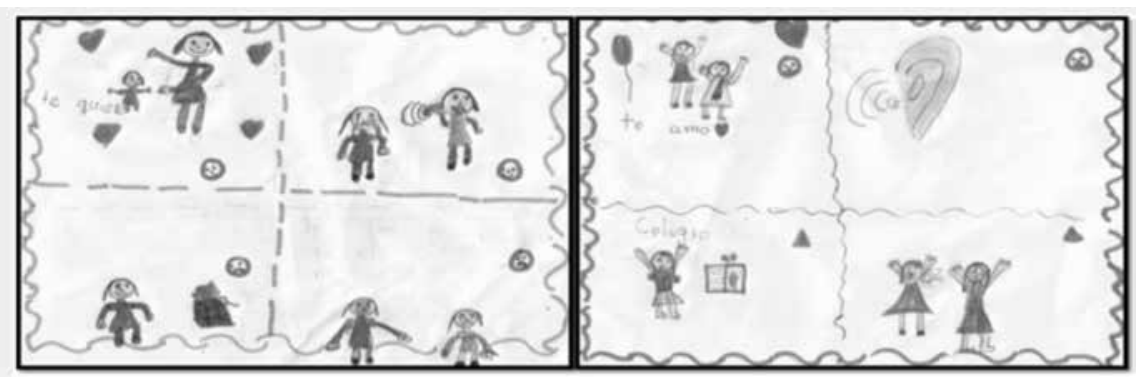

Fuente: niños y niñas de los grados cuarto y quinto de la institución educativa

Primero se les explicó a los niños, niñas y jóvenes cómo realizar los mapas, teniendo en cuenta que estos son una forma de expresar y plasmar realidades vividas, relaciones establecidas en el contexto escolar; luego se acordaron de manera grupal los símbolos, las convenciones (gráficas) que se tendrían en cuenta en la elaboración de cada uno de los mapas, los cuales permitieron tener una mayor comprensión de la problemática objeto de estudio, así como reconocer las relaciones existentes entre los actores de la experiencia.

Diseño y elaboración de los mapas: superada la fase explicativa, se inició la elaboración de los mapas en hojas de gran tamaño permitiendo la participación e interacción constante del grupo, utilizando las convenciones establecidas acorde a las categorías de reconocimiento (emocional, jurídico y social), menosprecio (maltrato, violencia, desprecio y desposesión) y justicia social. El objetivo era que los niños, niñas y jóvenes plasmaran sus vivencias, concepciones e ideas sobre las categorías presentes en su entorno escolar y familiar y su incidencia en las relaciones interpersonales y sociales.

Explicación de cada uno de los mapas: los estudiantes argumentaron de manera detallada aquello que plasmaron en los mapas y que evidencian las dinámicas presentes en las interrelaciones a nivel familiar, social y comunitario, que favorecían las formas de reconocimiento en los cuales podían ver garantizados sus derechos, su dignidad y su integridad; así como identificar situaciones que hacen evidente los modos de menosprecio que afectan la autorrealización, la autoimagen y la autoestima.

Para hacer más dinámica y participativa la exposición se nombró un relator o monitor de grupo, quien se encargaba de recopilar las reflexiones que luego socializaron ante el colectivo. Estas reflexiones fueron posteriormente analizadas por las investigadoras.

Acuerdos de transformación: con base en las reflexiones, los estudiantes elaboraron acuerdos grupales que promovieran prácticas de 
reconocimiento de sí mismo y de sus semejantes como sujetos de derechos para la construcción de paz con miras a una verdadera justicia social al interior de los centros educativos. En esta fase se interpretaron las vivencias, concepciones e imaginarios plasmados en los relatos y mapas frente al maltrato, la violencia y las humillaciones como modos de menosprecio, así como las formas de reconocimiento presentes en sus relaciones y que de alguna manera se convierten en el punto de transformación y el cambio requerido.

Análisis de los mapas y memoria de la cartografía: las investigadoras analizaron la información obtenida de los mapas con sus convenciones e interpretaron los escritos y relatos de los estudiantes. Los resultados se presentaron ante los niños, las niñas y los jóvenes para dar a conocer las dinámicas sociales que se viven al interior de sus familias y la institución educativa. A partir de la sistematización y la utilización de la cartografía social se confrontaron los postulados sobre el reconocimiento y el menosprecio expuestos en la teoría de Axel Honnet.

\section{Resultados}

La experiencia pedagógica "Sujetos Constructores de Convivencia Pacífica desde el Reconocimiento" evidenció que el nivel de conflicto escolar había aumentado y se presentaban conductas de maltrato, abuso de poder constante y agresividad dando cuenta de un bajo nivel de justicia social y la presencia de diversos modos de menosprecio que requerían la búsqueda de estrategias pedagógicas orientadas al reconocimiento de los estudiantes como sujetos de derechos y logrando su participación directa en la eliminación de dichas conductas para propiciar la convivencia pacífica, la participación democrática y la construcción de equidad que se requiere en una institución formadora de maestros, quienes serán los encargados de educar la sociedad del mañana.

La investigación permitió identificar que los niños, niñas y jóvenes que sufren menosprecio en las primeras etapas de su vida manifiestan dificultad para relacionarse con sus pares, convirtiéndose esto en una constante; ellos actúan con menosprecio por el otro, es decir, menospreciar al otro lo asumen como un acto válido y lo integran psicológicamente a su personalidad. Por tanto, los actos de violencia y maltrato vividos en la infancia influyen en el desarrollo de la personalidad (psiquis) y, en el mismo sentido, en el carácter (conductas). Así, un niño menospreciado recurrentemente integra el menosprecio a su psiquis y luego lo hace acto humano desde su carácter.

En la institución educativa se evidencian las formas de reconocimiento expuestas por Axel Honnet. En cuanto al reconocimiento emocional: se identificó que los estudiantes requieren un acompañamiento de los padres de familia y docentes que les permita manifestar sus opiniones, sentimientos, deseos propios y de los 
demás para aceptar o expresar diferencias de manera respetuosa a la luz de la dignidad humana. Sobre el reconocimiento jurídico: los estudiantes comprenden que es necesario exigir sus derechos y establecer límites en su actuar cuando estos vulneran los derechos de los demás. Con relación al reconocimiento social: los estudiantes construyen acciones de reparación en situaciones de vulnerabilidad y violencia.

La sistematización de experiencias al trabajar el campo social permite recopilar e interpretar historias de vida contadas desde la perspectiva de los actores, poniendo al descubierto realidades no visibilizadas que afectan el desarrollo de las comunidades y que les impiden su crecimiento personal, social, económico y cultural. Es así que la cartografía social posibilitó hacer una exploración, seguimiento e interpretación de las vivencias de los niños, niñas y jóvenes en su contexto familiar, escolar y comunitario que permitió entender las formas de actuar de las comunidades y la huella que van dejando a lo largo de su vida las interrelaciones con sus semejantes, evidenciando los niveles de exclusión a los cuales han estado tradicionalmente expuestos, ya que sus entornos fueron permeados por la cultura e idiosincrasia campesina, fruto de la violencia intergeneracional vivida en el municipio de Saboyá en décadas pasadas.

Actualmente los conflictos logran resolverse de manera pacífica utilizando el diálogo como punto de partida y reflexionando sobre lo ocurrido para tomar las mejores decisiones que favorezcan el ejercicio de los derechos humanos. El trabajo en equipo posibilita asumir responsabilidades de manera individual, garantizando el éxito del colectivo del cual hace parte, y esa interacción entre los diferentes actores genera relaciones de empatía, compañerismo y colaboración, favoreciendo aprendizajes en todos los niveles.

La construcción de paz desde las aulas está estrechamente relacionada con los vínculos sociales que establecen los diferentes integrantes de la comunidad, en contraposición a las relaciones excluyentes, desiguales y violentas que se deben evitar a través de la generación de espacios sanos de convivencia. Actuar de forma pacífica requiere un proceso de cambio de mentalidad, sentimientos y actitudes necesarios para cimentar las formas de reconocimiento como base de la dignidad del ser humano, permitiendo ver lo positivo en el otro y valorar sus capacidades, asumiendo que, como personas íntegras, debemos reconocer que en la diferencia podemos hallar el punto de encuentro en el que la solidaridad, la confianza y el respeto nos permitan crecer como sociedad.

\section{Conclusiones}

El proyecto de reconocimiento desde la justicia social ha de tener un manejo interdisciplinario en las instituciones educativas, de tal forma que permita abordar proyectos transversales como democracia y derechos humanos, tiempo libre, educación para sexualidad y construcción de ciudadanía, estilos de vida saludable, educación ambiental y cátedra de paz, con el fin de lograr que todas 
acciones escolares estén encaminadas a nutrir prácticas justas basadas fundamentalmente en la equidad, solidaridad, respeto por la dignidad y la justicia para promover la paz y la inclusión.

Es necesario ampliar y potenciar la implementación de la experiencia a las demás instituciones educativas para que exista un verdadero proceso de transformación del contexto familiar, social y cultural de la comunidad con el fin de que, a través del trabajo colaborativo y cooperativo, se fortalezcan lazos que les permitan generar acciones de mejoramiento que atiendan al bien común y creen espacios de sana convivencia que contribuyan a la construcción de paz.

La divulgación y promoción de esta experiencia permite redireccionar las prácticas de convivencia y construcción de paz, que hasta el momento se han desarrollado en las instituciones educativas, hacia la implementación de estrategias inclusivas y diferenciales que promuevan el reconocimiento de sí mismo como sujeto de derechos, buscando que la resolución pacífica de los conflictos potencie la construcción de procesos de convivencia democrática que permitan la reconstrucción del tejido social y sea posible encontrar el camino hacia la generación de la paz que requiere el país.

Es importante que las diferentes entidades gubernamentales de orden municipal, regional y nacional adelanten inversiones de orden social que permitan disminuir las brechas entre las comunidades y se logre una mayor equidad en la adquisición y goce de las garantías que poseen los seres humanos en condiciones de igualdad. Así mismo, es importante que se involucren y apoyen las iniciativas encaminadas al fortalecimiento de las formas reconocimiento y la superación de los modos de menosprecio como una manera efectiva de mejorar los ambientes escolares, para que los aprendizajes en las instituciones educativas sean de calidad.

\section{Referencias}

Antonio, B. (2011). Justicia social y equidad escolar. Una revisión actual. Revista Internacional de Educación para la Justicia Social (RIEJS),1(1), 9-45. Recuperado de https://revistas.uam.es/riejs/article/view/308/310

Arias, M. (2016) Erase un Colegio [Mensaje de un Blog]. Recuperado de https:// eraseuncolegio.wordpress.com/el-colegio/pactos-de-aula/

Díaz, M. V., y González, D. M. (2015). Experiencias de reconocimiento vividas por los niños en sus familias. Revista Colombiana de Ciencias Sociales, 6(2). Recuperado de http://www.funlam.edu.co/revistas/index.php/RCCS/ article/view/1444

Escudero, J., y Martínez, B. (2012). Las políticas de lucha contra el fracaso escolar: ¿programas especiales o cambios profundos del sistema y la educación? 
Revista de Educación, número extraordinario, 174-193. Recuperado de http:// www.carei.es/archivos_materiales/Escudero\%20y\%20Martinez\%202012.pdf

Escuela Normal Superior de Saboyá (18 de enero de 2016). Escuela Normal Superior de Saboyá. Recuperado de http://www.normaldesaboya.edu.co/ index.php/nuestra-institucion

Fernández, M, Ávila, A., y Taylos, H. (2007). SIG-P y experiencias de cartografía social en la ciudad de Bogotá (Colombia). Bogotá: UPZ Los Libertadores. Recuperado de http://www.observatoriogeograficoamericalatina.org.mx/ egal12/Nuevastecnologias/Sig/42.pdf

Gobernación del Huila (24 de septiembre de 2015). Áreas y Proyectos del Plan de Estudios. Recuperado de http://www.huila.gov.co/plan-de-estudios/2043educacion/pagina-educadores.html

Habegger, S., y Lulia, M. (2007). Cartografía Social. Recuperado de https://juanherrera.files.wordpress.com/2008/01/cartografia-social.pdf

Honnet, A. (1992). Integridad y desprecio: Motivos básicos de una concepción de la moral. ISEGORÍA. Revista de Filosofía Moral y Política, 5, 78-92.

Honnet, A. (1993). La Lucha por el Reconocimiento. Barcelona: Crítica.

Honnet, A. (2006a). El Reconocimiento como Ideología. Isegoría, 35, 129-150.

Honnet, A. (2006b). Redistribución como Reconocimiento. Respuesta a Nancy Fraser. En N. Fraser y A. Honneth, ¿Redistribución o reconocimiento? Madrid: Morata.

Honneth, A. (2010) Reconocimiento y menosprecio, sobre la fundamentación normativa de una teoría social. Buenos Aires: Katz Editores.

Honnet, A. (2011). La sociedad del Desprecio. Madrid: Trotta.

Jimenez, F. (2012). Conocer para comprender la violencia: origen, causas y realidad. Convergencia, 58(19). Recuperado de http://www.scielo.org.mx/ scielo.php?script=sci_arttext\&pid=S1405-14352012000100001

Martínez-Otero, V. (2005). Conflictividad Escolar y Fomento de la Convivencia. Revista Iberoamericana, 38. Recuperado de http://www.rieoei.org/rie38a02.htm

Matijasevic, M. T. (2015). Experiencias de Reconocimiento y Menosprecio en Campesinas y Campesinos de caldas. (Tesis de Maestría). Centro de Estudios Avanzados en Niñez y Juventud. Manizales. Recuperado de http://ridum. umanizales.edu.co:8080/xmlui/bitstream/handle/6789/2178/Tesis\%20 M.T.Matijasevic.pdf?sequence=1

Melgar, L. (2015). El arte de enseñar. Bloomington: Palibrio. Recuperado de https:// books.google.com.co/books?id=PyNyCQAAQBAJ\&pg=PT11\&lpg=PT11\&d 
$\mathrm{q}=\% 22 \mathrm{la}+\mathrm{educaci} \% \mathrm{C} 3 \% \mathrm{~B} 3 \mathrm{n}+$ no+cambia+al+mundo:+cambia+a+las+persona $\mathrm{s}+$ que+van+a+cambiar+el+mundo. $\% 22+$ paulo+freire\&source=bl\&ots $=\mathrm{aOTJ}$ m0FYx\&sig=mvXCt5i_52jhWPWWl6Y62u9eEE4\&hl=es-419\&sa=X\&ved=0a

Quintero, M., y Rojas, N. (2016). Desdibujando los territorios: Orientación para la elaboración cartografias sociales. Bogotá: World Vision.

Rawls, J. (1971). Teoría de la Justicia Social. Cambridge: Harvard University Press. Recuperado de https://etikhe.files.wordpress.com/2013/08/john_ rawls_-_teoria_de_la_justicia.pdf

Tovar, C. (2003). Reconocimiento de Derechos: Una Reflexión Ética. Lima. Recuperado de www.memoriayprofecia.com.pe/sites/default/files/Reflex_Tovar.doc 